\title{
The ACID PHOSPHATASE 1 regulates Pi stress adaptation by maintaining intracellular Pi homeostasis
}

\author{
Su Deng ${ }^{1}$, Jingyi $\mathrm{Li}^{1}$, Zezhen $\mathrm{Du}^{1}$, Zixuan $\mathrm{Wu}^{1}$, Jian Yang ${ }^{2}$, Hongmei Cai ${ }^{1}$, Gaobing $\mathrm{Wu}^{1}$, \\ Fangsen XU ${ }^{1}$, Yichao Huang ${ }^{3}$, Sheliang Wang ${ }^{1}$, and Chuang Wang ${ }^{1}$ \\ ${ }^{1}$ Huazhong Agricultural University \\ ${ }^{2}$ Sichuan University \\ ${ }^{3}$ Anhui Medical University
}

September 25, 2021

\begin{abstract}
The concentration and homeostasis of intracellular phosphate $(\mathrm{Pi})$ are crucial for sustaining cell metabolism and growth. During short-term Pi starvation, intracellular Pi is maintained relatively constant at the expense of vacuolar Pi. After the vacuolar stored $\mathrm{Pi}$ is exhausted, the plant cells induce the synthesis of intracellular acid phosphatase (APase) to recycle Pi from expendable organic phosphate (Po). In this study, the expression, enzymatic activity and subcellular localization of ACID PHOSPHATASE 1 (OsACP1) were determined. OsACP1 expression is specifically induced in almost all cell types of leaves and roots under Pi stress conditions. OsACP1 encodes an acid phosphatase with broad Po substrates and localizes in the endoplasmic reticulum (ER) and Golgi apparatus (GA). Phylogenic analysis demonstrates that OsACP1 has a similar structure with human acid phosphatase PHOSPHO1. Overexpression or mutation of OsACP1 affected Po degradation and utilization, which further influenced plant growth and productivity under both Pi-sufficient and Pi-deficient conditions. Moreover, overexpression of OsACP1 significantly affected intracellular Pi homeostasis and Pi starvation signalling. We concluded that OsACP1 is an active acid phosphatase that regulates rice growth under Pi stress conditions by recycling Pi from Po in the ER and GA.
\end{abstract}

\section{Hosted file}

Revise 1.docx available at https://authorea.com/users/341668/articles/538665-the-acidphosphatase-1-regulates-pi-stress-adaptation-by-maintaining-intracellular-pi-homeostasis

\section{Hosted file}

data-R1.pdf available at https://authorea.com/users/341668/articles/538665-the-acidphosphatase-1-regulates-pi-stress-adaptation-by-maintaining-intracellular-pi-homeostasis 